\title{
Gesture Recognition Based on Human Grasping Activities Using PCA-BMU
}

\author{
Nazrul H. ADNAN \\ Kolej Kemahiran Tinggi Mara (KKTM), \\ Mukim Serom 4 \& 5, 84410 Ledang, Johor \\ Darul Takzim, MALAYSIA
}

\author{
Mahzan T. \\ Kolej Kemahiran Tinggi Mara (KKTM), \\ Mukim Serom 4 \& 5, 84410 Ledang, Johor \\ Darul Takzim, MALAYSIA
}

\begin{abstract}
This research study presents the recognition of fingers grasps for various grasping styles of daily living. In general, the posture of the human hand determines the fingers that are used to create contact between an object at the same time while developing the touching contact. Human grasping can detect by studying the movement of fingers while bending during object holding. Ten right-handed subjects are participated in the experiment; each subject was fitted with a right-handed GloveMAP, which recorded all movement of the thumb, index, and middle of human fingers while grasping selected objects. GloveMAP is constructed using flexible bend sensors placed back of a glove. Based on the grasp human taxonomy by Cutkosky, the object grasping is distinguished by two dominant prehensile postures; that is, the power grip and the precision grip. The dataset signal is extracted using GloveMAP, and all the signals are filtered using Gaussian filtering method. The method is capable to improving the amplitude transmission characteristic with the minimal combination of time and amplitude response. The result was no overshoot in order to smoothen the grasping signal from unneeded signal (noise) that occurs on the input / original grasping data. Principal Component Analysis - Best Matching Unit (PCA-BMU) is a process of justifying the human grasping data involves several grasping groups and forming a component identified as nodes or neuron.
\end{abstract}

Keywords—recognition; grasp; grasp taxonomy; human finger; dimensionality reduction

\section{INTRODUCTION}

There are too many applications in this era that related to the human gesture that is include parts of the human body such as hands, face, body and many more. The hand gesture is one of the famous gestures used in daily life. People use the hand gesture to enhance the communication with others to deliver the information of thoughts effectively. This hand gesture will give a lot of important information of fingers or hand movement that can implement in the industrial applications such as video games industry, biomedical instrument, sports science, surveillance systems and many more. A device known as a Dataglove is presented as a medium to measure the information gain from hand or fingers activities. Dataglove is known as cyber glove or wired glove, and it is a device that can be donned by human. Any of physical data can capture by this technology using various sensors such as bending sensor or mostly known as flexible bend sensor, a force sensor or force resistive sensor, a tactile sensor and other types of sensor. Dataglove is also known as parts of "Haptic Science", which is

Kolej Kemahiran Tinggi Mara Ledang (KKTM), Majlis Amanah Rakyat (MARA) give meaning as science of applying tactile sensation to human interaction through computer.

GloveMAP can recognize and classify human grasping of some selected objects (Ball, Cylinder, Pen, Key, Disc, Scissors, Pins and Paper). The principle of GloveMAP is based on the concept of human grasping activities which is considered basic in daily living and was studied for a decade [1][2]. GloveMAP exploits the advantage of flexible bend sensors attached on GloveMAP hand surface to capture finger movement information when the user performs grasping activities. GloveMAP outputs are in a form of voltage over time that varies when the surface of flexible bending sensor is bending. The bending of flexible bend sensor will alter the resistivity of the sensor. The grasping activities will produce a signal that represents the characteristic of the grasping objects. The output signal must be filtered to reduce the noise produced by the thermal motion of electrons. The output signal is term as human grasping data. In finalizing the grasping signal, Principal Component Analysis (PCA) is used to reduce the data redundancy. PCA generally functions as to reduce the dimensionality of dataset in which there are a large number of interrelated variables, while maintaining as much as possible in dataset changes [3][4].

This research paper is structured as follows: Section 2 addresses the literature review of the related researches to the several approaches, applications and problems of recognizing the fingers grasping force signal. Section 3 describes the methodologies of the system. Section 4 describes the experiment of the research. Section 5 will present the results and discussion. Finally, section 6 described the conclusions and proposing some possible future work.

\section{LITERATURE REVIEW}

Nowadays, computer and related technology has become so distinctive, Human Computer Interaction (HCI) is one of the associated technology. Since HCI has such a common phenomenon, there was a need to make it as seamless as possible so that it was close to the natural human-to-human interaction. However, one big hurdle that must be overcome in order to achieve this objective was the lack of human grasping perception in today's computers. If computers can recognize human grasping activities, not only the interaction becomes more natural and easy through improved HCI, but many other useful applications could be developed. For example, a rehabilitation system device can be developed to assist the elderly and highly disabled people. Intelligent tutoring systems 
can take into account by transforming the system into education site. Regarding to this, the system is capable to be a smart tutorial for those who wanted a system with smart application using virtually hand motion or fingers movement. Human hand can be fitted with intelligent devices that are able to transform the users' grasping motion into many applications. In the last decade, there were numerous literatures on grasping force analysis, grasping force optimization and grasping force stability. The key problems in the last twenty years were force analysis [5] and grip strength [6], usually problem occurs when multi-fingered grasping takes place. Generally speaking, advances in the recognition of human activities such as motion control [7], hand grasping [8][9] and robot grasping [10] are progressing. They are demonstrated using popular methods such as EMG [11], Dataglove [12][13][14][15], and humanoid hand [10].

According to [16], the direct kinematics of fingertips is used to grasp the objects. They also proposed the position and orientation as the best methodologies for the study. Meanwhile for the data reduction and classifier method for finger grasping data, [17] stated that PCA is the best reduction method especially for the motionless position synergy angle configuration of the physical posture and contour of human hand / fingers whilst grasping the object.
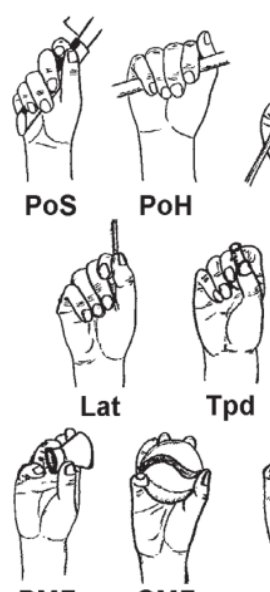

PMF CMF

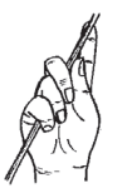

PoI

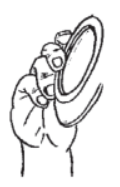

PoE

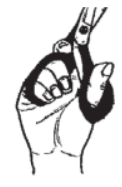

PoD
Fig. 1. Kamakura Grasp Taxonomy [2]

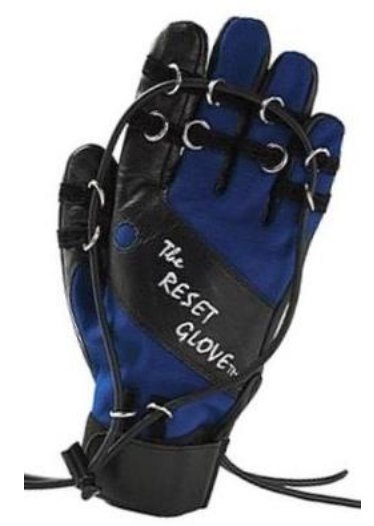

Fig. 2. Resistive interface glove [18]

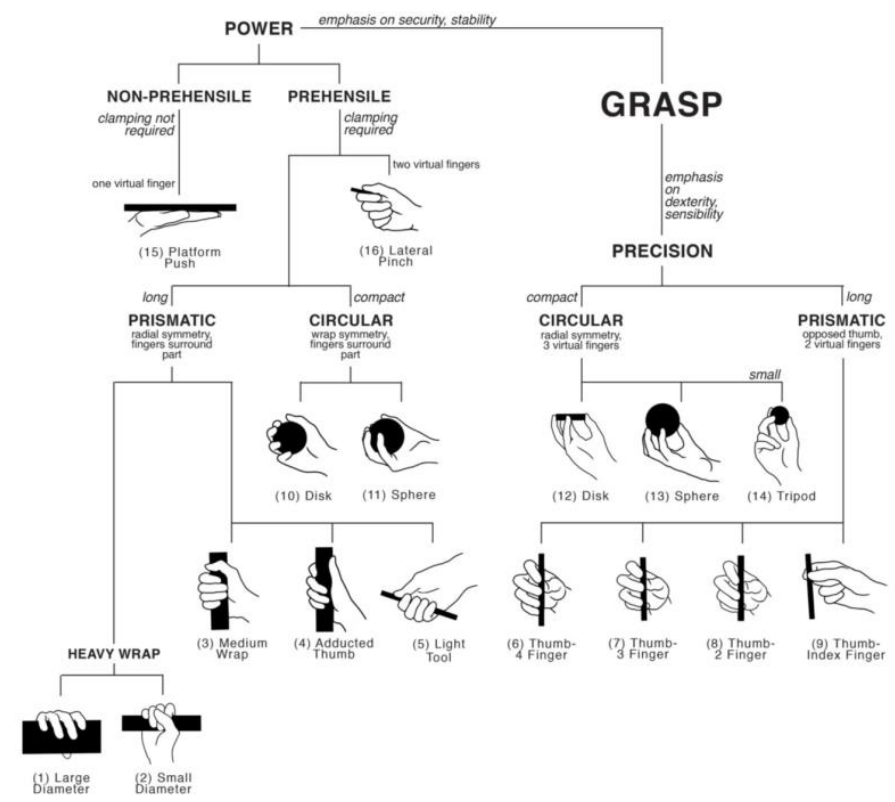

Fig. 3. Cutkosky Grasp Taxonomy [1]

Most of the research efforts on grasping taxonomy for the analysis of human grasping activities include the selection of experiment objects. According to Miller et al., to solve the grasping analysis in research by using grasp pre-shape which is defined as the finger configuration when a hand begins the fingers bending activities [19]. Napier, classified human hand shapes into a precision grasp and a power grasp [10]. In the power grasp, the object is grasped by the whole hand, including the fingers, thumb, and palm. In contrast, in the precision grasp, the object is grasped only by the fingertips. Kamakura et al., proposed an occupational therapist grasp taxonomy which consists of 14 hand shapes used in daily life [2]; this taxonomy is shown in Fig. 1. Fig. 2 shows the resistive interface glove which is used in conducting the experiment of human grasping activities. Meanwhile Fig. 3 illustrates the Cutkosky grasp taxonomy. Cutkosky, proposed the improved taxonomy on grasping activities using 16 hand shapes used by humans working with tools and metal parts [1]. Both taxonomies are identified as grasp types. Dataset of grasp preshapes for a human hand is produced from real human grasp activities.

\section{METHODOLOGY}

This section presents method used in characterizing fingers grasp capabilities for various grasping styles of daily living. In general, the posture of the human hand determines the fingers that are used to create contact between object at the same time develops the touching relation. The relationship between human grasp and selection of grasping object is based on the philosophy of grasping which is known as Cutkosky Grasp Taxonomy. The taxonomy distinguishes two dominant prehensile postures which are the power grip and the precision grip. According to Cutkosky (1989), all subjects should confine to single-handed operations and there should have been a better appreciation of how task requirements and object geometry combine to justify the grasp choice for better result of human grasp [1]. The next process flow is to eliminate or 
minimize the unwanted signal and noise by using Gaussian Filter. Gaussian Filtering makes grasping signal become smoother and lessens the abrupt changes in signal frequency. Then the grasping signals are analyzed using PCA. Since PCA functions as data reduction, PCA becomes the first choice method in reducing the redundancy in grasping signal. PCA is capable to generate an "Eigenfinger" for thumb, index and middle fingers of grasping data. Fig. 4 shows the example of fingers grasp testier and Fig. 5 shows a sample of human grasp object.

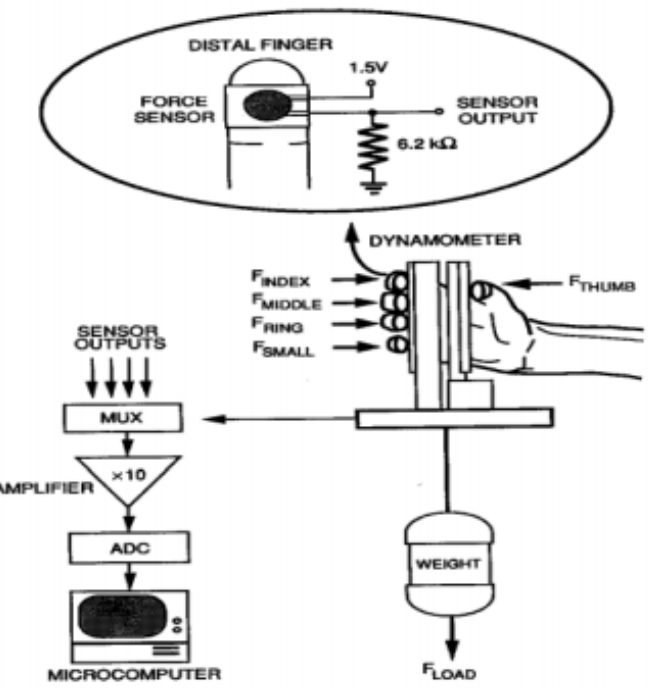

Fig. 4. Experiment of fingers grasps [20]

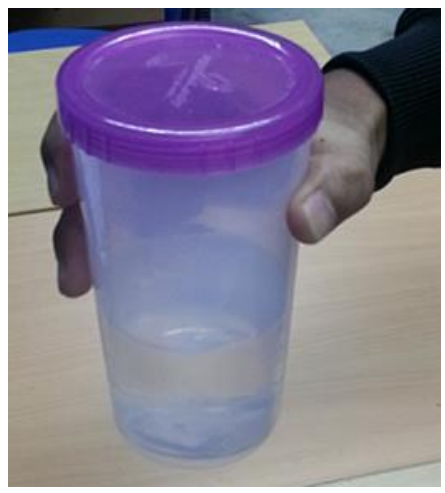

Fig. 5. Object grasping

\section{A. Gaussian Filtering Techniques}

GloveMAP signal is prepared with Gaussian filtering method in order to remove noise produced by random thermal motion of charge inside the electrical conductor. Noise within signal could affect the performance of objects' feature and classification. Resistors used in GloveMAP also would produce noise as heat inside resistors buildup. Each data collection from 8 objects will be filtered using Gaussian Filtering. Fig. 6(a) and Fig. 6(b) show unfiltered and filtered voltage produced from human grasping. Both figures demonstrate the result of Gaussian Filtering into raw voltage to reduce noises and overshoot. Gaussian has an advantage of reducing noises and overshoot of the input grasping signal.

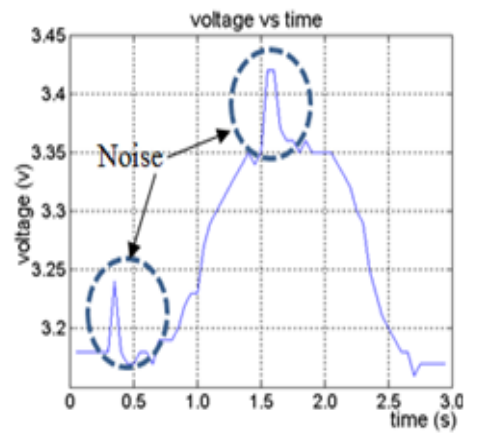

(a) (b)

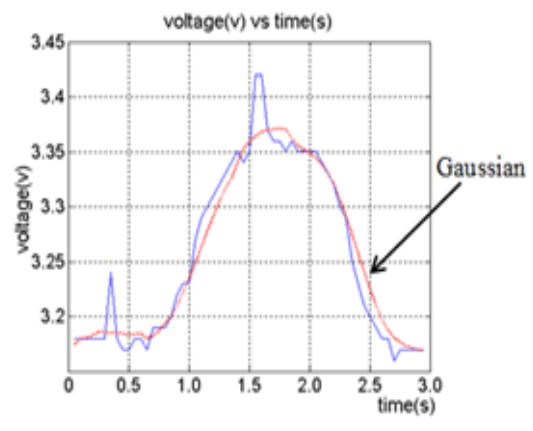

Fig. 6. a) Unfiltered voltage output with noise b) Voltage with Gaussian Filter

\section{B. Kinematical Finger Structure}

For the kinematical finger structure, finger joints consist of two main joints namely the Proximal Interphalangeal (PIP) and Interphalangeal (IP) joint of human finger. The other joint is the Metacarpophalangeal (MCP) joint. Fig. 7 illustrates a DIP flexion of human finger. Although the hand has so many flexions, the finger movement is highly constrained, below is the list of two motion finger constrains so that it cannot make arbitrary gestures:

1) Intrafinger constraints: This is the constraint between the joints of the same finger and the movement of joints can be approximated by the following equation:

$$
\theta_{D I P}=0.67 \theta_{P I P}
$$

Where $\theta_{D I P}$ known as the DIP bending angle meanwhile $\theta_{P I P}$ is the bending angle of the PIP joint.

2) Angle range constraints: This type of constraint refers to the limits of the ranges of finger motions as a result of hand anatomy. Fig. 8 shows the PIP flexion of human hand and it is usually within the following ranges.

$$
0^{0} \leq \theta_{P_{I P_{-} \text {Flexion }}} \leq\left(90^{\circ} \sim 100^{\circ}\right)
$$
hand.

and, meanwhile Fig. 9 shows the MCP flexion of human

$$
0^{0} \leq \theta_{\text {MCP_Flexion }} \leq 90^{\circ}
$$

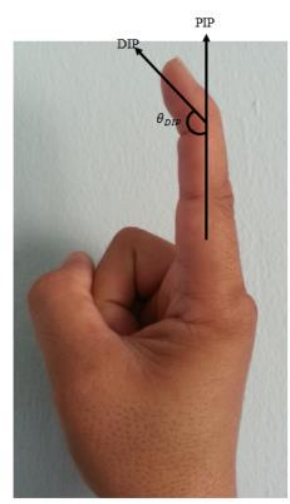

Fig. 7. DIP flexion 


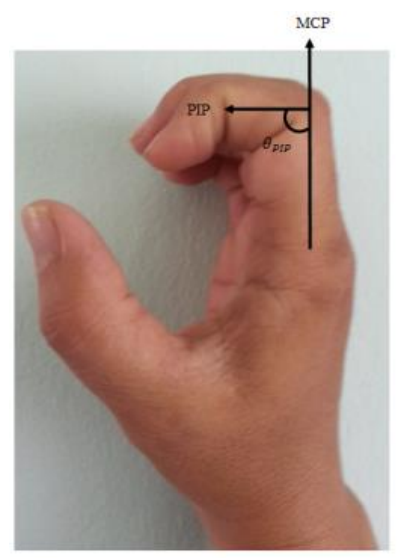

Fig. 8. PIP flexion

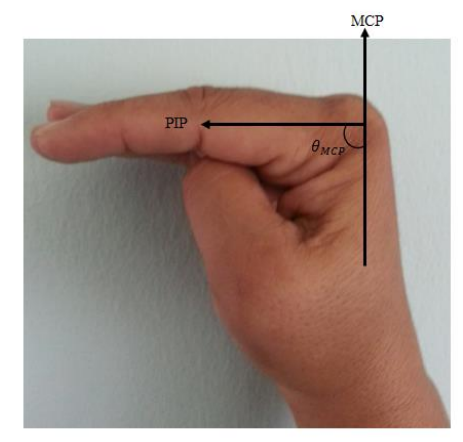

Fig. 9. MCP flexion

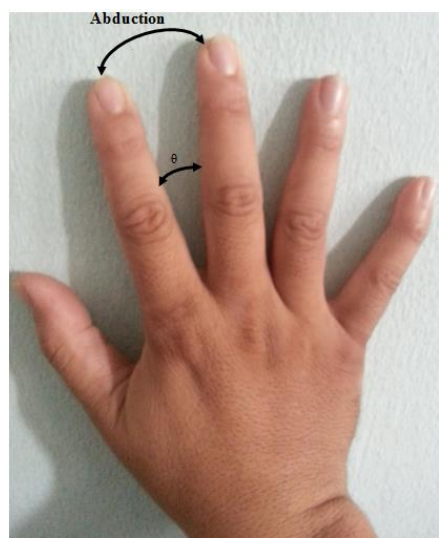

Fig. 10. Finger Abduction

Another kinematical finger movement is the finger abduction; Fig. 10 shows how fingers move in abduction action. Abduction is functioning by 2 conditions moving the index finger away from the middle finger or bringing the index finger close to the middle finger. Abduction also can be Thumb-Index, Thumb-Middle, or Index-Middle finger movement as shown in Fig. 11.

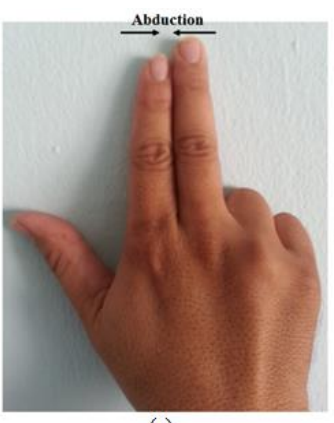

(a)

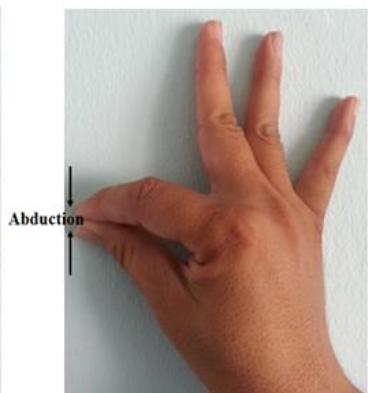

(b)

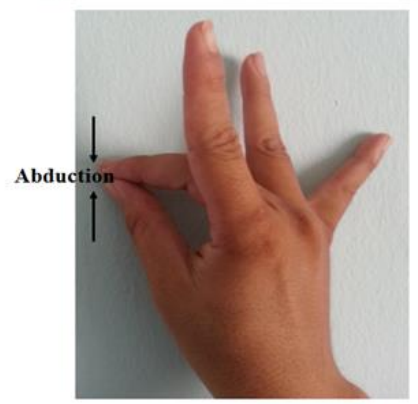

(c)

Fig. 11. Abduction (a) Index-Middle (b) Thumb-Index (c) Thumb-Middle

\section{Principal Components Analysis (PCA)}

PCA is found useful in many applications, but the basic application is the dimensionality reduction method. The grasping data signal could be calculated by converting the coordinate of the finger bending. It is defined as the space of Eigenfingers (feature spaces). For the example let the dataset, consisting of $p$ observation variables and $q$ observations for each variable stack into a matrix $X \epsilon R^{q \times p}$, with $q$ is column and $p$ is row of data and it is expressed in Equation (4):

$$
A=\left[\begin{array}{ccc}
x_{11} & x_{12} & x_{13} \\
x_{21} & x_{22} & x_{23} \\
\vdots & \vdots & \vdots \\
x_{q 1} & x_{q 2} & x_{q 3}
\end{array}\right]
$$

Where $A$ is a covariance matrix for 3 dimensional dataset, using usual dimension $x_{11}=\mathrm{x}$ dimension, $x_{12}=\mathrm{y}$ dimension and $x_{13}=\mathrm{z}$ dimension.

The principal component transform is defined as $A$ equal to the Eigenfingers matrix and defined as the roots of:

$$
\operatorname{determinant}(A-\lambda I)=|A-\lambda I|=0
$$

Where;

\section{$I=(q x p)$ identity matrix}

$\lambda=$ eigenvalue of $A$

Otherwise, $\lambda_{1}>\lambda_{2} \ldots>\lambda_{n}$ could be identified as the eigenvalues of the covariance (some other researchers termed this as the diagonal covariance matrix) of $A$. The analysis of PCA could be used by both Eigenfingers and Eigenvalues are 
requisite. Whereas Eigenvalues can be simplified as Eigenvalues $=$ Eigenfingers $*$ original data . Some analysis of the real numbers is dependent to both concepts (vectors and linear transformations). Eigenfingers $J$ of $A$ and Eigenvalues $\lambda$ can be determined as:-

$$
A_{J}=\lambda_{J}
$$

and simplified as:

$$
(A-\lambda I) X=0
$$

The concept of Jacobi method [21] is applied where $\lambda$ and $A$ were calculated and $I$ is known as the identity matrix. Lastly, it is simply to find the Eigenfingers determinant as shown in Equation (8).

$$
\operatorname{det}(A-\lambda I)=0
$$

\section{RESUlT AND DISCUSSION}

In this section, the analyses of overall step results are started accordingly from data acquisition, data analysis, features usage, and finally classifier recognition result.

\section{A. Human Grasping Data}

Fig. 12 shows the sample of 3 out of 8 objects grasping data. The figures show three main fingers results (thumb, index and middle) involved in the experiment. All figures show that middle and index finger were given more bending compared to the thumb. Basically index and middle fingers could be defined as the two strongest fingers meanwhile the thumb functioned as the main supportive finger whilst grasping the objects. Based on Fig. 12, it is proven that the signal for both fingers (index and middle finger) was more functioning compared to thumb fingers. Naturally, the thumb cannot bend more compared to the index and middle finger, however the thumb at the same time is moderately flexible (when the hand was spread, the thumb was easily standing a fair distance from the rest of the fingers).

\section{B. PCA-Best Matching Unit (PCA-BMU)}

The process of justifying the human grasping data involves several grasping groups and forming a component identified as nodes or neuron. The group of neurons basically has one main neuron located at the center of group of neurons, which is the winning neuron or centroid. Based on the explanation in the previous chapter, centroid or the winning neuron is formed by the competition of each neuron for representation of the group of data. The process of competing occurs until a next competing between other neuron except the centroid or winning neuron had been finalized.

To determine the PCA-Best Matching Unit (PCA-BMU) of the grasping data, the concept of neighborhood between neuron was applied. So, the next step was calculating all neurons nearby the centroid or winning neuron. One method to calculate the neighborhood between nodes and centroid or winning neuron is the Euclidean distance.

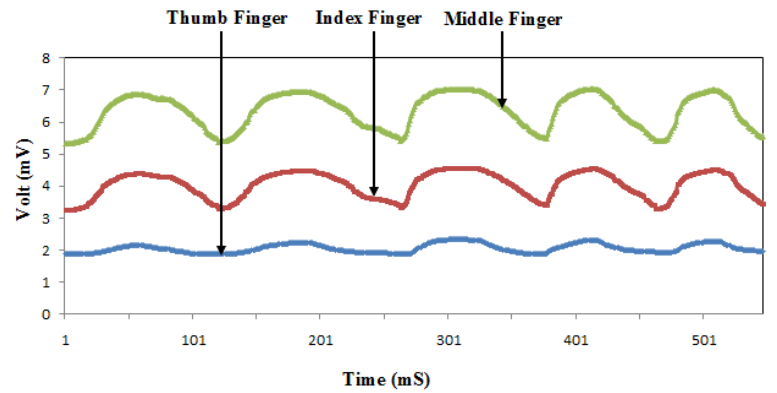

(a)

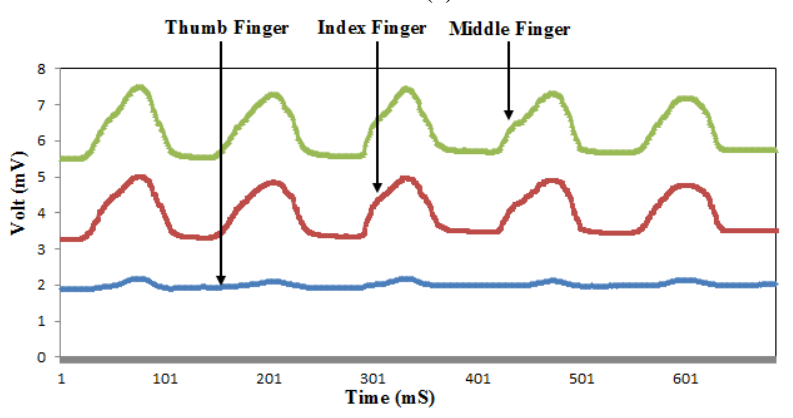

(b)

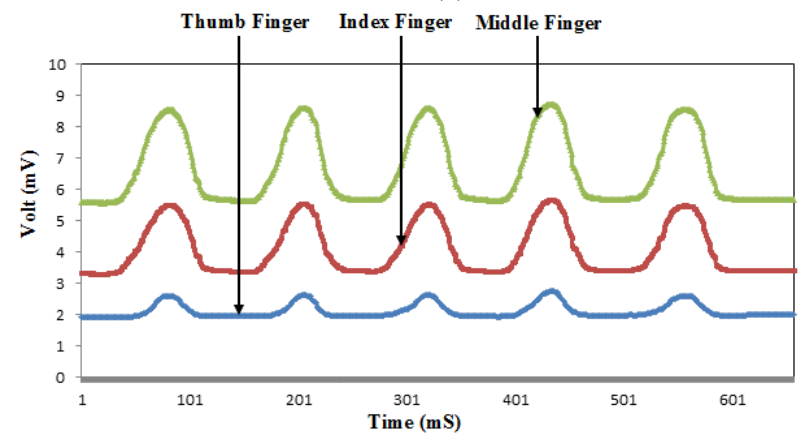

(c)

Fig. 12. Grasping signals of the object (a) "Ball” (b) "CD" (c) "Cylinder"

The steps to determine the nearest node to the winning neuron or centroid are stated below.

1) All nodes were justified using Euclidean Distance to winning neuron or centroid.

2) The equivalent or nearest node matching with any of the centroids were justified.

3) The nearest nodes to the winning neuron or centroid will form a group of node identified as "Cluster".

From a group of neuron, set of five nearest distances with a weight vector closest to the input vector of the centroid or winning neuron was tagged as the PCA-BMU. Lastly, to justify the features, the total sums of distance for all five nearest points were calculated and the result is known as PCA-BMU features. Fig. 13 to Fig. 15 show the PCA-BMU features with 5 nearest points for all objects. Meanwhile Table 1 to Table 3 shows the total sums of five nearest distance of object "Ball", "CD" and "Cylinder". 
(IJACSA) International Journal of Advanced Computer Science and Applications,

Vol. 6, No. 11, 2015

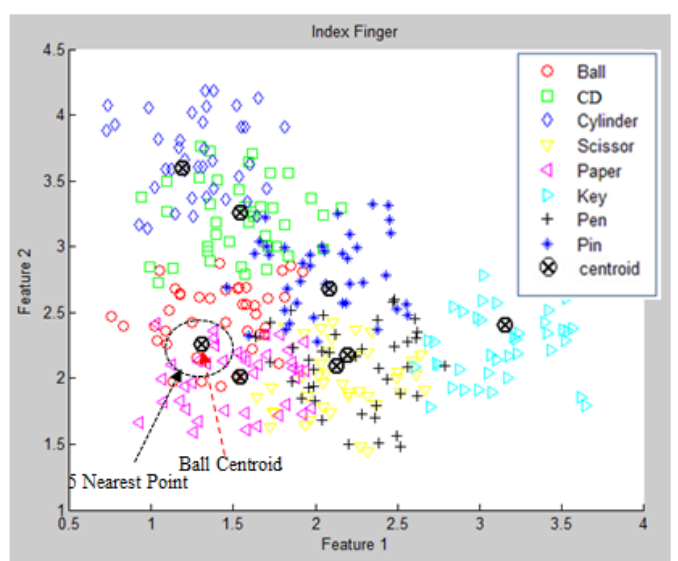

Fig. 13. 5 nearest points for PCA-BMU feature of the object "Ball"

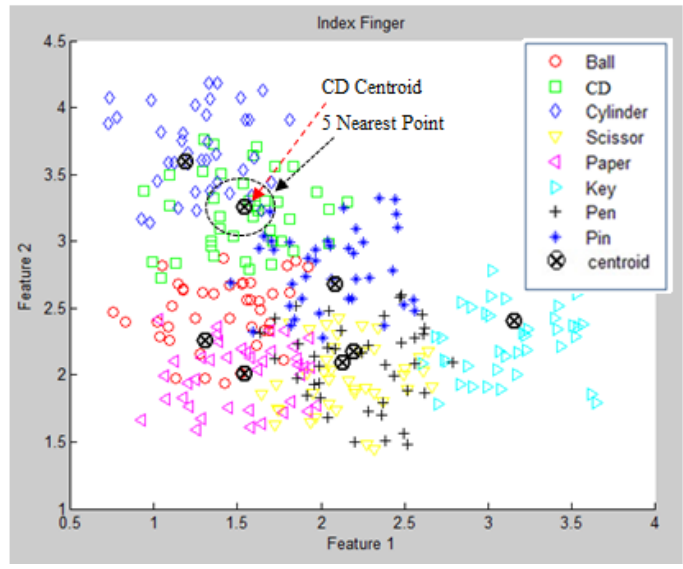

Fig. 14. 5 nearest points for PCA-BMU feature of the object "CD"

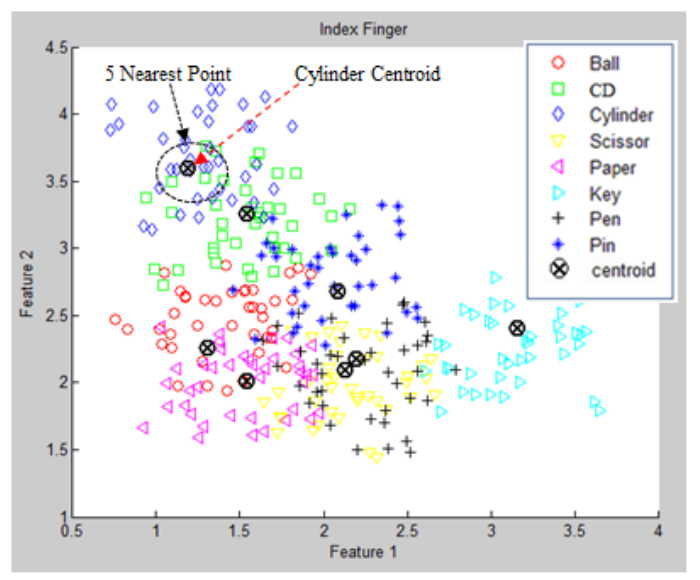

Fig. 15. 5 nearest points for PCA-BMU feature of the object "Cylinder"
TABLE I. SAMPLE OF 10 REPETITIONS GRASPING PERFORMANCES OF PCA-BEST MATCHING UNIT (PCA-BMU) FEATURES FOR OBJECT "BALL"

\begin{tabular}{|c|c|c|c|c|c|c|c|}
\hline \multirow{2}{*}{\multicolumn{2}{|c|}{ Ball Object }} & \multicolumn{5}{|c|}{5 nearest distance point } & \multirow{2}{*}{$\begin{array}{c}\text { PCA- } \\
\text { BMU } \\
\text { Features }\end{array}$} \\
\hline & & \multirow{2}{*}{$\begin{array}{c}1 \\
0.0482\end{array}$} & \multirow{2}{*}{$\begin{array}{c}\mathbf{2} \\
0.0901 \\
\end{array}$} & \multirow{2}{*}{$\begin{array}{c}3 \\
0.1111 \\
\end{array}$} & \multirow{2}{*}{$\frac{\mathbf{4}}{0.1202}$} & \multirow{2}{*}{$\begin{array}{c}\mathbf{5} \\
0.1213\end{array}$} & \\
\hline \multirow{3}{*}{$\begin{array}{c}\text { Repetition } \\
1\end{array}$} & Thumb & & & & & & 0.4910 \\
\hline & Index & 0.0815 & 0.0824 & 0.0823 & 0.0945 & 0.0956 & 0.4361 \\
\hline & Middle & 0.2746 & 0.2887 & 0.2938 & 0.3108 & 0.3215 & 1.4894 \\
\hline \multirow{3}{*}{$\begin{array}{c}\text { Repetition } \\
2\end{array}$} & Thumb & 0.1315 & 0.1382 & 0.1940 & 0.1968 & 0.3005 & 0.9610 \\
\hline & Index & 0.0673 & 0.0951 & 0.1166 & 0.1226 & 0.1245 & 0.5262 \\
\hline & Middle & 0.0840 & 0.0891 & 0.0912 & 0.0938 & 0.0954 & 0.4534 \\
\hline \multirow{3}{*}{$\begin{array}{c}\text { Repetition } \\
3\end{array}$} & Thumb & 0.0392 & 0.0733 & 0.0785 & 0.0847 & 0.885 & 0.3641 \\
\hline & Index & 0.1079 & 0.1200 & 0.1406 & 0.1582 & 0.2014 & 0.7281 \\
\hline & Middle & 0.0382 & 0.0608 & 0.0835 & 0.0840 & 0.0937 & 0.3602 \\
\hline \multirow{3}{*}{$\begin{array}{c}\text { Repetition } \\
4\end{array}$} & Thumb & 0.1080 & 0.1152 & 0.1154 & 0.1163 & 0.1165 & 0.5714 \\
\hline & Index & 0.0967 & 0.1072 & 0.1251 & 0.1594 & 0.1996 & 0.6879 \\
\hline & Middle & 0.0840 & 0.0938 & 0.0954 & 0.1001 & 0.1044 & 0.4776 \\
\hline \multirow{3}{*}{$\begin{array}{c}\text { Repetition } \\
5\end{array}$} & Thumb & 0.0197 & 0.0353 & 0.0429 & 0.0430 & 0.0440 & 0.1849 \\
\hline & Index & 0.0908 & 0.1266 & 0.1739 & 0.2977 & 0.3022 & 0.9912 \\
\hline & Middle & 0.1031 & 0.1061 & 0.1283 & 0.1498 & 0.1792 & 0.6666 \\
\hline \multirow{3}{*}{$\begin{array}{c}\text { Repetition } \\
6\end{array}$} & Thumb & 0.0810 & 0.0909 & 0.1817 & 0.1846 & 0.1928 & 0.731 \\
\hline & Index & 0.0434 & 0.0459 & 0.0486 & 0.0611 & 0.0688 & 0.2678 \\
\hline & Middle & 0.3403 & 0.3484 & 0.3555 & 0.3709 & 0.3815 & 1.7966 \\
\hline \multirow{3}{*}{$\begin{array}{c}\text { Repetition } \\
7\end{array}$} & Thumb & 0.0864 & 0.1008 & 0.1021 & 0.1193 & 0.1303 & 0.5389 \\
\hline & Index & 0.1404 & 0.1894 & 0.1929 & 0.1999 & 0.2011 & 0.9237 \\
\hline & Middle & 0.0811 & 0.0811 & 0.1097 & 0.1097 & 0.1201 & 0.5017 \\
\hline \multirow{3}{*}{$\begin{array}{c}\text { Repetition } \\
8\end{array}$} & Thumb & 0.0864 & 0.1008 & 0.1021 & 0.1193 & 0.1303 & 0.5389 \\
\hline & Index & 0.1448 & 0.1489 & 0.1737 & 0.2080 & 0.2100 & 0.8854 \\
\hline & Middle & 0.0520 & 0.0892 & 0.1079 & 0.1095 & 0.1095 & 0.4681 \\
\hline \multirow{3}{*}{$\begin{array}{c}\text { Repetition } \\
9\end{array}$} & Thumb & 0.0520 & 0.0534 & 0.0565 & 0.0593 & 0.0687 & 0.2899 \\
\hline & Index & 0.0315 & 0.1041 & 0.1182 & 0.1515 & 0.2593 & 0.6646 \\
\hline & Middle & 0.1380 & 0.1711 & 0.2279 & 0.2454 & 0.2683 & 1.0507 \\
\hline \multirow{3}{*}{$\begin{array}{c}\text { Repetition } \\
10\end{array}$} & Thumb & 0.0864 & 0.1008 & 0.1021 & 0.1193 & 0.1209 & 0.5295 \\
\hline & Index & 0.0971 & 0.1076 & 0.1250 & 0.1597 & 0.1992 & 0.6886 \\
\hline & Middle & 0.1090 & 0.1165 & 0.1297 & 0.1378 & 0.1502 & 0.6432 \\
\hline
\end{tabular}

TABLE II. SAMPLE OF 10 REPETITIONS GRASPING PERFORMANCES OF PCA-BEST MATCHING UNIT (PCA-BMU) FEATURES FOR OBJECT "CD"

\begin{tabular}{|c|c|c|c|c|c|c|c|}
\hline \multirow{2}{*}{\multicolumn{2}{|c|}{ CD Object }} & \multicolumn{5}{|c|}{5 nearest distance point } & \multirow{3}{*}{\begin{tabular}{|c|} 
PCA- \\
BMU \\
Features \\
$\mathbf{0 . 2 0 3 4}$
\end{tabular}} \\
\hline & & \multirow{2}{*}{$\frac{1}{0.0201}$} & \multirow{2}{*}{$\frac{2}{0.0240}$} & \multirow{2}{*}{$\frac{3}{0.0434}$} & \multirow{2}{*}{$\frac{4}{0.0518}$} & \multirow{2}{*}{$\frac{5}{0.0641}$} & \\
\hline \multirow{3}{*}{$\begin{array}{c}\text { Repetition } \\
1\end{array}$} & Thumb & & & & & & \\
\hline & Index & 0.1177 & 0.1773 & 0.1819 & 0.1983 & 0.1996 & 0.8749 \\
\hline & Middle & 0.0431 & 0.0714 & 0.2177 & 0.2274 & 0.2428 & 0.8024 \\
\hline \multirow{3}{*}{$\begin{array}{c}\text { Repetition } \\
2\end{array}$} & Thumb & 0.0775 & 0.0873 & 0.0957 & 0.0962 & 0.1003 & 0.4571 \\
\hline & Index & 0.1581 & 0.1715 & 0.1726 & 0.2260 & 0.2538 & 0.9820 \\
\hline & Middle & 0.1190 & 0.1637 & 0.1865 & 0.2217 & 0.2634 & 0.9543 \\
\hline \multirow{3}{*}{$\begin{array}{c}\text { Repetition } \\
\mathbf{3}\end{array}$} & Thumb & 0.0957 & 0.0962 & 0.1003 & 0.1003 & 0.1024 & 0.4949 \\
\hline & Index & 0.1345 & 0.1426 & 0.1665 & 0.2176 & 0.2356 & 0.8967 \\
\hline & Middle & 0.0915 & 0.2628 & 0.2793 & 0.2856 & 0.2938 & 1.2130 \\
\hline \multirow{3}{*}{$\begin{array}{c}\text { Repetition } \\
4\end{array}$} & Thumb & 0.0599 & 0.0703 & 0.0839 & 0.0919 & 0.0957 & 0.4016 \\
\hline & Index & 0.2548 & 0.2581 & 0.2745 & 0.2828 & 0.2928 & 1.3629 \\
\hline & Middle & 0.1140 & 0.1534 & 0.1538 & 0.1678 & 0.2781 & 0.8672 \\
\hline \multirow{3}{*}{$\begin{array}{c}\text { Repetition } \\
5\end{array}$} & Thumb & 0.0957 & 0.0962 & 0.1003 & 0.1003 & 0.1024 & 0.4949 \\
\hline & Index & 0.1859 & 0.2417 & 0.3167 & 0.3460 & 0.3534 & 1.4437 \\
\hline & Middle & 0.0398 & 0.1496 & 0.1590 & 0.1793 & 0.1985 & 0.7262 \\
\hline \multirow{3}{*}{$\begin{array}{c}\text { Repetition } \\
6\end{array}$} & Thumb & 0.0323 & 0.0443 & 0.0698 & 0.0788 & 0.0920 & 0.3172 \\
\hline & Index & 0.1153 & 0.1748 & 0.1854 & 0.1947 & 0.1997 & 0.8699 \\
\hline & Middle & 0.0424 & 0.0700 & 0.1117 & 0.1131 & 0.1156 & 0.4528 \\
\hline \multirow{3}{*}{$\begin{array}{c}\text { Repetition } \\
7\end{array}$} & Thumb & 0.0323 & 0.0331 & 0.0443 & 0.0698 & 0.0788 & 0.2583 \\
\hline & Index & 0.1382 & 0.1419 & 0.1743 & 0.2027 & 0.2339 & 0.891 \\
\hline & Middle & 0.1117 & 0.1131 & 0.1156 & 0.1166 & 0.1213 & 0.5783 \\
\hline \multirow{3}{*}{$\begin{array}{c}\text { Repetition } \\
8\end{array}$} & Thumb & 0.0989 & 0.1012 & 0.1026 & 0.1043 & 0.1043 & 0.5113 \\
\hline & Index & 0.1304 & 0.1830 & 0.2015 & 0.3182 & 0.3353 & 1.1684 \\
\hline & Middle & 0.1090 & 0.1117 & 0.1131 & 0.1149 & 0.1156 & 0.5643 \\
\hline \multirow{3}{*}{$\begin{array}{c}\text { Repetition } \\
9\end{array}$} & Thumb & 0.0989 & 0.1012 & 0.1026 & 0.1043 & 0.1043 & 0.5113 \\
\hline & Index & 0.2092 & 0.2174 & 0.2244 & 0.2344 & 0.2456 & 1.131 \\
\hline & Middle & 0.1059 & 0.1166 & 0.1213 & 0.1253 & 0.1328 & 0.6019 \\
\hline \multirow{3}{*}{$\begin{array}{c}\text { Repetition } \\
10\end{array}$} & Thumb & 0.0902 & 0.0989 & 0.1012 & 0.1026 & 0.1043 & 0.4972 \\
\hline & Index & 0.1991 & 0.2086 & 0.3261 & 0.3572 & 0.3608 & 1.4518 \\
\hline & Middle & 0.0390 & 0.1467 & 0.1523 & 0.1551 & 0.1556 & 0.6487 \\
\hline
\end{tabular}


TABLE III. SAMPLE OF 10 REPETITIONS GRASPING PERFoRMANCES OF PCA-Best MATChING UNIT (PCA-BMU) FEATURES FOR OBJECT "CYLINDER"

\begin{tabular}{|c|c|c|c|c|c|c|c|}
\hline \multirow{2}{*}{\multicolumn{2}{|c|}{ Cylinder Object }} & \multicolumn{5}{|c|}{5 nearest distance point } & \multirow{2}{*}{$\begin{array}{c}\text { PCA- } \\
\text { BMU } \\
\text { Features }\end{array}$} \\
\hline & & \multirow{2}{*}{$\begin{array}{c}1 \\
0.0774\end{array}$} & \multirow{2}{*}{$\begin{array}{c}2 \\
00819\end{array}$} & \multirow{2}{*}{$\begin{array}{c}\mathbf{3} \\
00940\end{array}$} & \multirow{2}{*}{$\begin{array}{c}\mathbf{4} \\
0.0957\end{array}$} & \multirow{2}{*}{$\begin{array}{c}5 \\
0.0962\end{array}$} & \\
\hline & Thumb & & & & & & 0.4451 \\
\hline Repetition & Index & 0.2201 & 0.2541 & 0.3065 & 0.3624 & 0.3660 & 1.5090 \\
\hline & Middle & 0.0342 & 0.0634 & 0.1072 & 0.1237 & 0.1244 & 0.4529 \\
\hline \multirow{3}{*}{$\begin{array}{c}\text { Repetition } \\
2\end{array}$} & Thumb & 0.0228 & 0.0421 & 0.0643 & 0.0742 & 0.0934 & 0.2968 \\
\hline & Index & 0.1540 & 0.1896 & 0.2205 & 0.2792 & 0.3114 & 1.1546 \\
\hline & Middle & 0.0506 & 0.0525 & 0.0970 & 0.1693 & 0.1863 & 0.5557 \\
\hline \multirow{3}{*}{$\begin{array}{c}\text { Repetition } \\
3\end{array}$} & Thumb & 0.0957 & 0.0962 & 0.0980 & 0.1003 & 0.1003 & 0.4905 \\
\hline & Index & 0.0453 & 0.1442 & 0.1976 & 0.1985 & 0.2476 & 0.8332 \\
\hline & Middle & 0.0426 & 0.0591 & 0.1002 & 0.1227 & 0.1772 & 0.5018 \\
\hline \multirow{3}{*}{$\begin{array}{c}\text { Repetition } \\
4\end{array}$} & Thumb & 0.0558 & 0.0593 & 0.0597 & 0.0637 & 0.0703 & 0.3088 \\
\hline & Index & 0.0840 & 0.1022 & 0.1481 & 0.1972 & 0.2568 & 0.7883 \\
\hline & Middle & 0.0418 & 0.1043 & 0.1175 & 0.1273 & 0.2167 & 0.6077 \\
\hline \multirow{3}{*}{$\begin{array}{c}\text { Repetition } \\
5\end{array}$} & Thumb & 0.1024 & 0.1082 & 0.1082 & 0.1096 & 0.1096 & 0.5379 \\
\hline & Index & 0.0683 & 0.0881 & 0.1011 & 0.1076 & 0.1633 & 0.5285 \\
\hline & Middle & 0.0478 & 0.0479 & 0.0501 & 0.0525 & 0.0576 & 0.2560 \\
\hline \multirow{3}{*}{$\begin{array}{c}\text { Repetition } \\
6\end{array}$} & Thumb & 0.0931 & 0.0948 & 0.0970 & 0.0989 & 0.1012 & 0.485 \\
\hline & Index & 0.1626 & 0.2519 & 0.2697 & 0.3068 & 0.3410 & $\begin{array}{l}0.705 \\
1.332\end{array}$ \\
\hline & Middle & 0.0390 & 0.0482 & 0.0706 & 0.1071 & 0.1453 & 0.4102 \\
\hline \multirow{3}{*}{$\begin{array}{c}\text { Repetition } \\
7\end{array}$} & Thumb & 0.0055 & 0.0133 & 0.0556 & 0.0675 & 0.0717 & 0.2136 \\
\hline & Index & 0.1515 & 0.1861 & 0.2201 & 0.2798 & 0.3076 & 1.1451 \\
\hline & Middle & 0.0495 & 0.0521 & 0.0953 & 0.1589 & 0.1605 & 0.5163 \\
\hline \multirow{3}{*}{$\begin{array}{c}\text { Repetition } \\
8\end{array}$} & Thumb & 0.0455 & 0.0948 & 0.0970 & 0.0989 & 0.1012 & 0.4374 \\
\hline & Index & 0.0423 & 0.0587 & 0.0986 & 0.1208 & 0.1746 & 0.495 \\
\hline & Middle & 0.0863 & 0.1175 & 0.1334 & 0.1461 & 0.1551 & 0.6384 \\
\hline \multirow{3}{*}{$\begin{array}{c}\text { Repetition } \\
9\end{array}$} & Thumb & 0.0578 & 0.0948 & 0.0970 & 0.0989 & 0.0997 & 0.4482 \\
\hline & Index & 0.0519 & 0.0776 & 0.1101 & 0.1224 & 0.1886 & 0.5506 \\
\hline & Middle & 0.0654 & 0.0723 & 0.0815 & 0.0942 & 0.0946 & 0.408 \\
\hline \multirow{3}{*}{$\begin{array}{c}\text { Repetition } \\
10\end{array}$} & Thumb & 0.0436 & 0.0521 & 0.0528 & 0.0649 & 0.0928 & 0.3062 \\
\hline & Index & 0.0524 & 0.0702 & 0.0958 & 0.1537 & 0.2783 & 0.6504 \\
\hline & Middle & 0.0373 & 0.0556 & 0.0889 & 0.0913 & 0.0925 & 0.3656 \\
\hline
\end{tabular}

\section{CONCLUSION AND FUTURE WORK}

In this paper, we proposed the method to classify human grasping signal for several selected objects which based on PCA-BMU techniques. The chosen of both PCA and Best Matching Unit (BMU) for this research capable to generating the best method to smoothen up the grasp signal of features. Experimental results show that the both method works well in defining grasp signal with only a usage of few principal components and also capable to identifying the grasp type of an input motion data. For next plan works, the results are by adding the signal processing technique in the research. This signal processing employ in solving the problem of analyzing more sophisticated signal pattern especially on the signal produced during transition gesture and continuing gesture

\section{ACKNOWLEDGMENT}

Special thanks to all members of KKTM Ledang and MARA for providing the research equipment's and internal foundations. This work was supported by the Majlis Amanah Rakyat (MARA).

\section{REFERENCES}

[1] Cutkosky, Mark R. "On grasp choice, grasp models, and the design of hands for manufacturing tasks." Robotics and Automation, IEEE Transactions on 5, no. 3 (1989): 269-279.

[2] Kamakura, N., Matsuo, M., Ishii, H., Mitsuboshi, F., \& Miura, Y. "Patterns of static prehension in normal hands", American Journal of Occupational Therapy, 34(7) (1980), 437-445.

[3] Cobos, S., Ferre, M., Angel Sanchez-Uran, M., Ortego, J., \& Aracil, R. "Human hand descriptions and gesture recognition for object manipulation".Computer methods in biomechanics and biomedical engineering, 13(3) (2010), 305-317.

[4] Turk, M., \& Pentland, A. "Eigenfaces for recognition". Journal of cognitive neuroscience, 3(1) (1991), 71-86.
[5] Xu, Jijie, Michael Y. Wang, Hong Wang, and Zexiang Li. "Force analysis of whole hand grasp by multifingered robotic hand." In Robotics and Automation, 2007 IEEE International Conference on, pp. 211-216. IEEE, 2007.

[6] McDowell, Thomas W., Bryan M. Wimer, Daniel E. Welcome, Christopher Warren, and Ren G. Dong. "Effects of handle size and shape on measured grip strength." International Journal of Industrial Ergonomics 42, no. 2 (2012): 199-205.

[7] Capisani, Luca Massimiliano, and Antonella Ferrara. "Trajectory planning and second-order sliding mode motion/interaction control for robot manipulators in unknown environments." Industrial Electronics, IEEE Transactions on 59, no. 8 (2012): 3189-3198.

[8] Kerr, Jeffrey, and Bernard Roth. "Analysis of multifingered hands." The International Journal of Robotics Research 4, no. 4 (1986): 3-17.

[9] Song, D., Huebner, K., Kyrki, V., \& Kragic, D. "Learning task constraints for robot grasping using graphical models". In Intelligent Robots and Systems (IROS), 2010 IEEE/RSJ International Conference on (pp. 1579-1585). IEEE.

[10] Tsoli, Aggeliki, and Odest Chadwicke Jenkins. "Neighborhood denoising for learning high-dimensional grasping manifolds." In Intelligent Robots and Systems, 2008. IROS 2008. IEEE/RSJ International Conference on, pp. 3680-3685. IEEE, 2008.

[11] Cipriani, Christian, Franco Zaccone, Silvestro Micera, and M. Chiara Carrozza. "On the shared control of an EMG-controlled prosthetic hand: analysis of user-prosthesis interaction." Robotics, IEEE Transactions on 24, no. 1 (2008): 170-184.

[12] Park, Jongwoo, and Joono Cheong. "Analysis of collective behavior and grasp motion in human hand." In Control Automation and Systems (ICCAS), 2010 International Conference on, pp. 2514-2518. IEEE, 2010.

[13] Adnan, Nazrul H., Khairunizam Wan, A. B. Shahriman, S. K. Zaaba, Shafriza nisha Basah, Zuradzman M. Razlan, D. Hazry, M. Nasir Ayob, M. Nor Rudzuan, and Azri A. Aziz. "Measurement of the flexible bending force of the index and middle fingers for virtual interaction". Procedia Engineering 41 (2012): 388-394.

[14] Häger-Ross, Charlotte, and Marc H. Schieber. "Quantifying the independence of human finger movements: comparisons of digits, hands, and movement frequencies." The Journal of neuroscience 20, no. 22 (2000): 8542-8550.

[15] Todorov, Emanuel, and Zoubin Ghahramani. "Analysis of the synergies underlying complex hand manipulation." In Engineering in Medicine and Biology Society, 2004. IEMBS'04. 26th Annual International Conference of the IEEE, vol. 2, pp. 4637-4640. IEEE, 2004.

[10] Napier, John R. "The prehensile movements of the human hand." Journal of bone and joint surgery 38, no. 4 (1956): 902-913.

[11] Iberall, Thea. "Human prehension and dexterous robot hands." The International Journal of Robotics Research 16, no. 3 (1997): 285-299.

[12] Tortora, Gerard J., and Sandra Reynolds Grabowski. "Principles of anatomy and physiology." (1993).

[13] Chao, Edmund Y. Biomechanics of the hand: a basic research study. World Scientific, 1989.

[14] Ratnasingam, Sivalogeswaran, and T. M. McGinnity. "Object recognition based on tactile form perception." In Robotic Intelligence In Informationally Structured Space (RiiSS), 2011 IEEE Workshop on, pp. 26-31. IEEE, 2011.

[15] Burke, David., S. C. Gandevia, and G. Macefield. "Responses to passive movement of receptors in joint, skin and muscle of the human hand."The Journal of physiology 402, no. 1 (1988): 347-361.

[16] Cobos, S., Ferre, M., Angel Sanchez-Uran, M., Ortego, J., \& Aracil, R. "Human hand descriptions and gesture recognition for object manipulation". Computer methods in biomechanics and biomedical engineering, 13(3) (2010), 305-317.

[17] Jerde, T. E., Soechting, J. F., \& Flanders, M. "Biological constraints simplify the recognition of hand shapes". Biomedical Engineering, IEEE Transactions on, 50(2) (2003), 265-269.

[18] Reference from Fishpond Ltd., 2015

[19] Miller, A. T., Knoop, S., Christensen, H. I., \& Allen, P. K. “Automatic grasp planning using shape primitives". In Robotics and Automation, 
2003. Proceedings. ICRA'03. IEEE International Conference on (Vol. 2, pp. 1824-1829). IEEE.

[20] Radwin, Robert G., Seoungyeon Oh, Todd R. Jensen, and John G. Webster. "External finger forces in submaximal five-finger static pinch prehension."Ergonomics 35, no. 3 (1992): 275-288.
[21] Jacobi, J., Fraser, G. L., Coursin, D. B., Riker, R. R., Fontaine, D., Wittbrodt, E. T., ... \& Lumb, P. D. "Clinical practice guidelines for the sustained use of sedatives and analgesics in the critically ill adult". Critical care medicine, 30(1) (2002), 119-141. 